\title{
Medium response from mini-jets and in-medium hadronization in relativistic heavy ion collisions
}

\author{
Sangwook Ryu ${ }^{a, b, *}$ Scott McDonald, ${ }^{c}$ Chun Shen, ${ }^{a, d}$ Sangyong Jeon ${ }^{c}$ and Charles \\ Gale $^{c}$ \\ ${ }^{a}$ Department of Physics, Wayne State University, \\ 666 W Hancock Street, Detroit, MI 48201, United States \\ ${ }^{b}$ Institut für Theoretische Physik, Goethe-Universität Frankfurt (FIAS), \\ Max-von-Laue-Str. 1, 60438 Frankfurt am Main, Germany \\ ${ }^{c}$ Department of Physics, McGill University, \\ 3600 rue University, Montreal, Quebec H3A 2T8, Canada \\ ${ }^{d}$ RIKEN BNL Research Center, Brookhaven National Laboratory, Upton, NY 11973, USA
}

E-mail: sangwook.ryu@wayne.edu

In ultra-relativistic heavy-ion collisions, partons with intermediate energy (5-10) GeV excite ripples as they travel through the Quark-Gluon Plasma (QGP). These ripples' effect on particle production phenomenology is not very well studied. In this work, we extend our previous work and present study using a state-of-the-art hybrid framework that consists of the IP-Glasma pre-thermalization dynamics, viscous hydrodynamics (MUSIC), jet energy loss (MARTINI), and hadronic re-scatterings (UrQMD). This framework consistently handles both soft and hard physics involved in relativistic heavy ion collisions. The energy loss of mini-jets, whose energy is between 5 to $10 \mathrm{GeV}$, and their induced medium excitation are considered. The energy and momentum lost from the mini-jets are treated as either free-streaming wave-packets or causal diffusive perturbations of the hydrodynamic flow. They alter the energy-stress tensor evaluated on the particlization hypersurface during the hadronization as jet induced medium response. In addition, this approach uses a novel in-medium fragmentation, where the color neutrality of a string is enforced locally. We quantify the importance of the medium response from mini-jets propagation and their in-medium hadronization on identified particle $p_{T}$-spectra and their flow anisotropic coefficients.

HardProbes 2020

1-6 June 2020

Austin, Texas

${ }^{*}$ Speaker 


\section{Introduction}

High-energy nucleus-nucleus collisions have played crucial roles in studying QCD matter at high temperature, provided that ultra-relativistic heavy ion experiments are where we have partons with large occupation number. The hybrid framework, which combines viscous hydrodynamics and hadronic cascade, provides a quantitative description of a wide range of soft (low- $p_{T}$ ) observables in heavy ion collisions [1,2]. In the meantime, jet-medium interactions, which are quantified as the nuclear modification factor, also turned out to be useful probes. The energy loss of high- $p_{T}$ jets, as well as the medium response due to jet quenching have been studied using comprehensive MonteCarlo frameworks [3]. While the low- $p_{T}$ and high- $p_{T}$ regimes have been extensively explored, the intermediate- $p_{T}$ regime, where contributions from the bulk QGP medium and mini-jets are comparable to each other, remains a challenging topic. Given that the mini-jet contribution can affect the intermediate and low- $p_{T}$ observables and a proper consideration of mini-jets is necessary for better understanding of QGP, we extend our previous work [4] such that mini-jet energy loss and diffusive medium response are incorporated into the hybrid framework. We present effects and theoretical uncertainties of different treatments of energy-loss and hadronization of mini-jets.

\section{Framework}

In this work, the bulk medium evolution is modeled by the IP-Glasma + MUSIC + UrQMD hybrid approach [5]. We use a equation of state (EoS) based on lattice QCD calculation from the hotQCD Collaboration [6]. The shear viscosity to entropy ratio is set to be $\eta / s=0.13$, while the temperature-dependent bulk viscosity is enhanced by factor of $4 / 3$ compared to that used in Ref. [1,2] because of the EoS update. In the dilute hadronic phase, fluid cells are convert to particles via the Cooper-Frye particlization prescription on a isothermal surface with $T_{\mathrm{sw}}=140 \mathrm{MeV}$. Viscous corrections from shear stress tensor and bulk viscous pressure at particlization are included.

To address the intermediate and higher $p_{T}$ regimes within our hybrid approach, it is necessary to model the production and energy loss of (mini-)jets. This is done by using MARTINI MonteCarlo event generator [7], which takes radiative and collisional energy losses into account. We simulate mini-jet production by QCD processes using PYTHIA 8 [8] where we have a minimum threshold $\hat{p}_{T, \text { min }}$ of the transverse momentum transfer in $2 \rightarrow 2$ partonic processes. The produced mini-jets are then followed by propagation through the QGP medium and energy loss.

To explore influence and uncertainties of the medium response to mini-jet energy loss, we consider two limiting cases for low-energy partons with their energy $E<4 T$ - free-streaming and diffusion. In the free-streaming approach, the low-energy partons are assumed to have no further interaction with the medium. They freely propagate to the hadronization surface. We refer this case as the weak coupling limit. In the opposite strongly-coupled limit, the medium response due to mini-jet energy loss is modeled by relativistic diffusion of particle density current. Time evolution of the parton density is determined by the casual diffusion equation

$$
\left(\frac{\partial}{\partial t}-\tau_{D} \bar{c}_{\perp}^{2} \nabla_{x_{\perp}}^{2}-\tau_{D} \bar{c}_{\|}^{2} \frac{\partial^{2}}{\partial x_{\|}^{2}}+\tau_{D} \frac{\partial^{2}}{\partial t^{2}}\right) \rho_{x}\left(t, \mathbf{x}_{\perp}, x_{\|}\right)=0,
$$

where we assume the effective speed of second sound $\bar{c}_{\perp}$ in the transverse direction is different from $\bar{c}_{\|}$in the longitudinal direction. This assumption takes into account different expansion rates 
in the transverse and longitudinal directions. Those effective speeds are evaluated for each energy loss instance, based on its trajectory. On average $\bar{c}_{\perp}$ and $\bar{c}_{\|}$are respectively 0.7 and 0.6. Once we have instances of mini-jet energy loss depositing energy/momentum $q_{\hat{j}}^{\mu}$, the linear response to the energy-stress tensor $\delta T_{i}^{\mu \nu}$ on the $i$-th element of discretized particlization hypersurface has the form of

$$
\begin{aligned}
\delta T^{\mu v}\left(x_{i}\right)=\sum_{\hat{j}} \delta T_{\hat{j}}^{\mu v}=\sum_{\hat{j}} & \rho_{x}\left(s_{\mathrm{fin}, \hat{j}}+\delta x_{i \hat{j}}^{\alpha} u_{\alpha}\left(x_{\mathrm{fin}, \hat{j}}\right), \delta \mathbf{x}_{i \hat{j}}\right) \\
& \quad \times u^{\beta}\left(x_{i}\right) u_{\beta}\left(x_{\mathrm{fin}, \hat{j}}\right)\left[\frac{q_{\hat{j}}^{\mu} q_{\hat{j}}^{v}}{q_{\hat{j}}^{0}}+Q_{\hat{j}}\left(u^{\mu} u^{\nu}\left(x_{i}\right)-\frac{P_{\mathrm{sw}}}{\epsilon_{\mathrm{sw}}} \Delta^{\mu v}\left(x_{i}\right)\right)\right],
\end{aligned}
$$

where the summation goes over all the energy-loss instances. The $s_{\text {fin }, \hat{j}}$ is time elapsed in the rest frame of fluid, while the $\hat{j}$-th instance of energy loss propagates with flow velocity down to the space-time point $x_{\mathrm{fin}, \hat{j}}^{\mu}$ on the particlization hypersurface. The $\delta \mathbf{x}_{i \hat{j}}=x_{i}-x_{\mathrm{fin}, \hat{j}}$ is the displacement vector between the fluid cell $i$ and energy-momentum source $\hat{j}$. Finally, the value of $Q_{\hat{j}}$ is determined by requiring energy conservation on the hyper-surface, $q_{\hat{j}}^{0}=\int_{\Sigma} d^{3} \Sigma_{\nu} \delta T_{\hat{j}}^{0 v}$.

Once the mini-jet feedback are propagated to the particlization hyper-surface, they contribute to the local energy-stress tensor. It is possible to decompose $T^{\mu v}$ with response to obtain thermodynamic quantities, flow velocity, and viscous corrections,

$$
T^{\mu \nu}=T_{\mathrm{bg}}^{\mu \nu}+\delta T^{\mu \nu}=\epsilon u^{\mu} u^{\nu}-(P+\Pi)\left(g^{\mu \nu}-u^{\mu} u^{v}\right)+\pi^{\mu \nu} .
$$

It is then followed by the Cooper-Frye particlization sampling for transition to hadronic cascades.

As jet partons and those low energy partons in the weakly-coupled approach reach the hadronization surface at $T_{\text {final }}=175 \mathrm{MeV}$, we apply a new in-medium hadronization scheme based on fragmentation of strings formed by a pair of jet parton and thermal parton [9]. The color of the thermal partner is sampled according that of the jet parton to ensure local color neutrality. The string mass $M$ is sampled from the probability distribution $P(M) \propto M \exp \left(-M / T_{\text {final }}\right)$ [10]. Once the thermal partons are sampled, we subtract their energy-momentum currents from the hydrodynamic background to avoid double counting their contribution in the Cooper-frye particlization.

\section{Results and Discussion}

The hadron chemistry from the string fragmentation and Cooper-Frye particlization are different. Therefore, the measurements of $p / \pi$ ratios at intermediate $p_{T}$ regions are important observables to elucidate the nature of hadronization in a hot and dense environment.

Figure 1 shows the identified hadrons $p_{T}$-spectra and the ratio of protons to pions in $\mathrm{Pb}$ $\mathrm{Pb}$ collisions at $\sqrt{s_{\mathrm{NN}}}=2.76 \mathrm{TeV}$. Both kinematics and chemistry of measured hadrons are sensitive to the treatment of medium response. In the weakly-coupled limit (free-streaming), all of the low-energy partons from the jet shower are hadronized with their thermal partners via the string fragmentation. Sampling these thermal partons results in more consumption of energy and momentum from the fluid background and reducing the particle production from the Cooper-Frye particlization. Because the string fragmentation produces more $\pi \mathrm{s}$ and less $p \mathrm{~s}$ than the thermal 

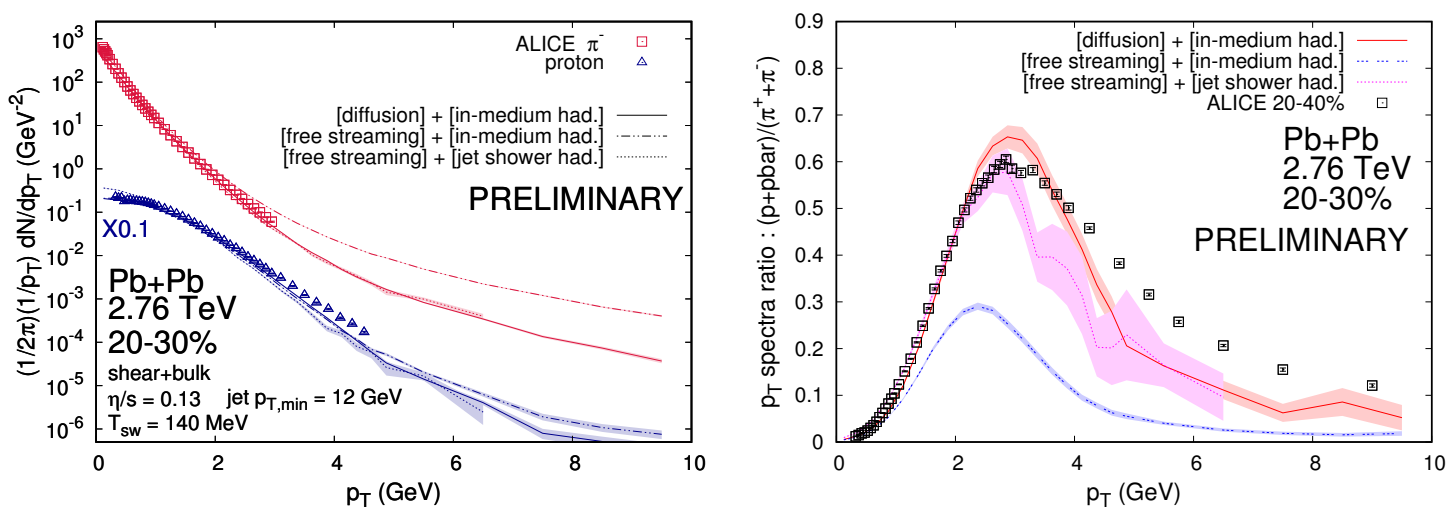

Figure 1: The identified hadrons $p_{T}$-spectra and their ratio with different treatments of mini-jet feedback and hadronization. $p_{T, \min }=12 \mathrm{GeV}$ and $\alpha_{S}=0.3$ for all cases. The ALICE data for the $p_{T}$ spectra [11] and ratio [12] are also shown for comparison.

equilibrium expectation, the $p / \pi$ ratios in this approach is largely suppressed and disfavored by the measurements as shown in Fig. 1. On the other hand, the strongly-coupled approach with diffusion can nicely reproduce the peak structure in the measured $p / \pi$ ratios. Therefore, our results favors that the low-energy partons from the jet shower are strongly interacting with the medium and hadronize according to thermal distributions, just like other thermal partons inside the bulk fluid. This calculation underestimated the $p / \pi$ ratio for $p_{T}>4 \mathrm{GeV}$ because the proton spectrum becomes too steep compared to the data. This discrepancy suggests the simulated hydrodynamic radial flow is somewhat weaker than that in the measurements.

For completeness, we also present a calculation with hadronizing the pure mini-jet shower without any thermal partons through the string fragmentation. In contrast to the in-medium hadronization, this pure jet-shower hadronization only ensures color neutrality on a global scale within an event. We find that particles from the string fragmentation start to have sizable contribution to the $p / \pi$ ratios for $p_{T}>2.5 \mathrm{GeV}$. For $p_{T}$ between 2.5 to $5 \mathrm{GeV}$, the $p / \pi$ ratios from the pure jet shower are $\sim 20 \%$ lower than those in the strongly-couple approach with in-medium hadronization. For $p_{T}>5 \mathrm{GeV}$, the identified particle spectra are dominated by the fragmentations of hard partons in the jet shower.

\section{Conclusion}

We present a hybrid theoretical framework, which incorporates both bulk medium and (mini)jets in a comprehensive manner. For bulk evolution, we have hydrodynamics with fluctuating prethermalization dynamics and hadronic microscopic transport to handle expanding fireball medium. For energetic partons, we have mini-jet energy loss as well as medium response. This unified framework enables us to study low and intermediate $p_{T}$ regimes simultaneously. We showed that distributions of thermal and mini-jet hadrons depend on the evolution of low-energy mini-jet partons and hadronization methods. Therefore, future systematic studies on the corresponding observables, including $p_{T}$-spectra and anisotropic flow coefficients in the intermediate- $p_{T}$ regime, will lead us to the better understanding of evolution and hadronization of mini-jets in heavy ion collisions. 


\section{Acknowledgements}

This work is partially funded by the Deutsche Forschungsgemeinschaft (DFG, German Research Foundation) - Project number 315477589 - TRR 211, and supported by the DAAD funded by BMBF (Project-ID 57314610). We thank Yasuki Tachibana and Abhijit Majumder for fruitful discussions. C. S. is supported in part by the U.S. Department of Energy (DOE) under grant number DE-SC0013460 and in part by the National Science Foundation (NSF) under grant number PHY-2012922. This work is supported in part by the Natural Sciences and Engineering Research Council of Canada and in part by the U.S. Department of Energy, Office of Science, Office of Nuclear Physics, within the framework of the Beam Energy Scan Theory (BEST) Topical Collaboration. Computation for this work was done on the Cedar cluster maintained by WestGrid and Compute Canada.

\section{References}

[1] S. Ryu, J. F. Paquet, C. Shen, G. S. Denicol, B. Schenke, S. Jeon and C. Gale, Phys. Rev. Lett. 115, no.13, $132301(2015)$

[2] S. McDonald, C. Shen, F. Fillion-Gourdeau, S. Jeon and C. Gale, Phys. Rev. C 95, no. 6, 064913 (2017)

[3] R. A. Soltz, [arXiv:2003.11728 [physics.data-an]].

[4] S. Ryu, S. McDonald, C. Shen, S. Jeon and C. Gale, PoS High-pT 2017, 014 (2019).

[5] B. Schenke, C. Shen and P. Tribedy, [arXiv:2005.14682 [nucl-th]].

[6] A. Bazavov et al. [HotQCD], Phys. Rev. D 90, 094503 (2014)

[7] B. Schenke, C. Gale and S. Jeon, Phys. Rev. C 80, 054913 (2009)

[8] T. Sjöstrand, S. Mrenna and P. Z. Skands, JHEP 0605, 026 (2006) ; Comput. Phys. Commun. 178, 852 (2008)

[9] S. Ryu, Ph.D. dissertation : Integrated description of heavy ion collisions at RHIC and the LHC, McGill University (2016)

[10] B. Andersson, G. Gustafson, G. Ingelman and T. Sjostrand, Phys. Rept. 97, 31-145 (1983)

[11] B. Abelev et al. [ALICE Collaboration], Phys. Rev. C 88, 044910 (2013)

[12] J. Adam et al. [ALICE], Phys. Rev. C 93, no.3, 034913 (2016) 